\title{
Seedling and Adult Plant Resistance to Powdery Mildew in Chinese Bread Wheat Cultivars and Lines
}

Z. L. Wang, Institute of Crop Breeding and Cultivation/National Wheat Improvement Center, Chinese Academy of Agricultural Sciences (CAAS), No. 12 Zhongguancun South Street, 100081, Beijing, China and Northwest Sci-Tech University of Agriculture and Forestry, Yangling, 712100, Shaanxi, China; L. H. Li, Institute of Crop Breeding and Cultivation/National Wheat Improvement Center, CAAS; Z. H. He, Institute of Crop Breeding and Cultivation/National Wheat Improvement Center, CAAS and CIMMYT China Office, C/O CAAS, No. 12 Zhongguancun South Street, 100081, Beijing, China; X. Y. Duan and Y. L. Zhou, Institute of Plant Protection, CAAS, No. 2 Yuanmingyuan West Road, 100094, Beijing, China; X. M. Chen, Institute of Crop Breeding and Cultivation/National Wheat Improvement Center, CAAS; M. Lillemo and R. P. Singh, International Maize and Wheat Improvement Center (CIMMYT), Apdo Postal 6-641, 06600, Mexico D.F., Mexico; H. Wang, Northwest Sci-Tech University of Agriculture and Forestry; and X. C. Xia, Institute of Crop Breeding and Cultivation/National Wheat Improvement Center, CAAS

\begin{abstract}
Wang, Z. L., Li, L. H., He, Z. H., Duan, X. Y., Zhou, Y. L., Chen, X. M., Lillemo, M., Singh, R. P., Wang, H., and Xia, X. C. 2005. Seedling and adult plant resistance to powdery mildew in Chinese bread wheat cultivars and lines. Plant Dis. 89:457-463.

Powdery mildew, caused by Blumeria graminis f. sp. tritici, is a widespread wheat disease in China. Identification of race-specific genes and adult plant resistance (APR) is of major importance in breeding for an efficient genetic control strategy. The objectives of this study were to (i) identify genes that confer seedling resistance to powdery mildew in Chinese bread wheat cultivars and introductions used by breeding programs in China and (ii) evaluate their APR in the field. The results showed that (i) 98 of 192 tested wheat cultivars and lines appear to have one or more resistance genes to powdery mildew; (ii) $P m 8$ and $P m 4 b$ are the most common resistance genes in Chinese wheat cultivars, whereas $P m 8$ and $P m 3 d$ are present most frequently in wheat cultivars introduced from CIMMYT, the United States, and European countries; (iii) genotypes carrying $P m 1, P m 3 e, P m 5$, and $P m 7$ were susceptible, whereas those carrying $P m 12, P m 16$, and Pm20 were highly resistant to almost all isolates of $B$. graminis f. sp. tritici tested; and (iv) 22 genotypes expressed APR. Our data showed that the area under the disease progress curve, maximum disease severity on the penultimate leaf, and the disease index are good indicators of the degree of APR in the field. It may be a good choice to combine major resistance genes and APR genes in wheat breeding to obtain effective resistance to powdery mildew.
\end{abstract}

Powdery mildew, caused by Blumeria graminis f. sp. tritici, is an important disease of wheat (Triticum aestivum) worldwide, especially in highly productive areas with a maritime or semicontinental climate (1). Losses in grain yield from 12 to $45 \%$ have been reported in the United States $(8,18,19,21)$. In China, powdery mildew on wheat occurred only occasionally in the southwestern plateau and coastal area of Shandong Province before the 1970s (23,31). However, its importance increased notably during the early 1980 s, mainly due

Corresponding authors: Z. H. He E-mail: zhhe@public3.bta.net.cn or X. C. Xia

E-mail: xiaxianchun@caas.net.cn

This research was supported by the international collaboration program of the National Natural Science Foundation of China (NSFC).

Accepted for publication 4 December 2004.

DOI: 10.1094/PD-89-0457

(C) 2005 The American Phytopathological Society to the high rates of nitrogenous fertilizer, production of semidwarf wheat cultivars, and expansion of irrigated areas. The area of wheat production affected by powdery mildew was 2.9 million ha in 1981. This increased to 12.0 million ha in 1990 , with the disease causing an estimated grain yield losses of 14.4 million tons (33).

Utilization of resistant cultivars is the most economical and environmentally safe means of controlling powdery mildew (1). To date, $48 \mathrm{Pm}$ genes or alleles at 32 loci are known to confer race-specific resistance in wheat $(14,16)$. Selection pressure exerted by cultivars that carry single racespecific genes results in a rapid build-up of the pathogen population possessing matching virulence genes and contributes to reducing resistance durability (28). Therefore, knowledge of the presence and frequency of virulence genes in the pathogen population is useful for replacing ineffective genes with effective ones. Information on resistance genes present in important wheat cultivars and knowledge of avirulence or virulence in pathogen populations is valuable for planning crosses in wheat breeding programs and for developing management strategies, including cultivar recommendations and deployment schemes $(15,19,34)$. Such information is also valuable to predict the breakdown of resistance genes caused by shifting pathogen populations.

Resistance to powdery mildew that retards infection, growth, and reproduction of the pathogen in adult plants, but not in seedlings, has been defined as slow mildewing (28) or adult plant resistance (APR; 5). This type of resistance can be identified in cultivars with defeated racespecific genes or cultivars that lack racespecific resistance genes (1). APR to powdery mildew is more durable than racespecific resistance. For example, cv. Knox and its derivatives (e.g., cv. Massey) have remained resistant to powdery mildew infection for over 20 years, despite being grown commercially on large areas $(9,28)$. Some wheat breeders are interested in using APR genes, and many genotypes with APR have been identified and used in wheat improvement $(4,9,36)$.

China is the largest wheat producer in the world, with about 25 million ha sown annually. The Chinese Academy of Agricultural Sciences has divided the country's wheat area into 10 agro-ecological zones based on wheat types and the response to biotic and abiotic stresses. Winter, facultative, and spring wheat types sown in both autumn and spring are grown; therefore, Chinese wheat germ plasm is very diverse in many aspects. However, little attention has been paid to investigating APR to powdery mildew and achieving durable resistance to Chinese isolates of $B$. graminis $\mathrm{f}$. sp. tritici. The objectives of our study were to (i) identify probable $P m$ genes that confer seedling resistance in Chinese wheat cultivars and various introductions frequently used by breeding programs in China and (ii) identify the presence of APR in these cultivars based on field trials.

MATERIALS AND METHODS

Isolates of $B$. graminis f. sp. tritici. Twenty isolates of $B$. graminis f. sp. tritici 
(Table 1) with different virulence patterns (7), collected from various regions of China, were used for identifying genes conferring resistance at the seedling stage. Isolate E20, with a broad spectrum of virulence, was used for evaluating APR to powdery mildew in field trials.

Wheat germplasm. Included in this study were 56 widely grown Chinese cultivars and 28 advanced breeding lines, as well as 108 cultivars and lines introduced from Europe, the United States, Brazil, and the International Maize and Wheat Improvement Center (CIMMYT) in Mexico (Tables 2 and 3). The cultivars and lines are available at the National Wheat Improvement Center of China upon request. Twenty differential cultivars and lines (Table 1) with known Pm genes $(16,24)$ were included in each test, and the low and high infection types displayed by them toward B. graminis f. sp. tritici isolates were used for postulating resistance genes in the wheat cultivars tested.

Seedling studies. In all, 192 wheat cultivars and advanced lines were evaluated using 20 B. graminis f. sp. tritici isolates in the greenhouse at the Institute of Plant Protection, Chinese Academy of Agricultural Sciences, in the spring of 1999 and 2003 according to the method described by Xiang et al. (35). Between five and seven plants of each cultivar and differential line were grown in flat plastic trays 70 by 45 by $18 \mathrm{~cm}$ in size. The highly susceptible check cv. Jingshuang 16 was planted randomly four times in each tray to evaluate uniformity of inoculum distribution. Inoculation with each of the 20 isolates was performed in separate trays, when the first leaves were fully expanded. Host reactions were recorded 7 to 10 days after inoculation, when the susceptible checks were heavily infected. A 0-to-4 infection type scale (29) was used for recording the host response to infection, where $0=$ no visible symptoms; $0 ;=$ hypersensitive necrotic flecks; $1=$ minute colonies with few conidia produced; $2=$ colonies with moderately developed hyphae, but few conidia; 3 = colonies with well-developed hyphae and abundant conidia, but colonies not joined together; and $4=$ colonies with well-developed hyphae and abundant conidia, and colonies mostly joined together.

Field trials. During the $2000-01$ and 2002-03 crop seasons, 52 and 76 winter wheat cultivars, respectively, were evaluated for APR to powdery mildew at the Institute of Crop Breeding and Cultivation, Chinese Academy of Agricultural Sciences. Thirty cultivars or lines were common to both experiments. Field trials were planted in a randomized complete block design with three replications; each plot consisted of two 2-m rows spaced $25 \mathrm{~cm}$ apart. Approximately 150 seed were sown in each plot. Spreader rows of the susceptible cv. Jingshuang 16 surrounded the test cultivars and lines and also were planted every 15 plots. The nursery was surrounded by triticale (Triticosecale Wittmack) to discourage contamination by other powdery mildew isolates. Inoculation was carried out with the isolate E20 of B. graminis f. sp. tritici prior to plants reaching stem elongation. The cultivar response to powdery mildew was scored for the first time 2 weeks after inoculation and then at weekly intervals until leaves were physiologically mature. The infection severity on 10 arbitrarily selected plants from each plot was recorded based on the 0-to- 9 scale described by Saari and Prescott (26). Powdery mildew severity on the penultimate leaf (= F-1 leaf ) was rated 2 weeks after flowering, based on the actual percentage of leaf area covered by powdery mildew, and then every 6 to 8 days.

A disease index (I) was calculated using the formula $\mathrm{I}=\left(0 \times n_{0}+1 \times n_{1}+\ldots\right.$ $\left.+9 \times n_{9}\right) / 9 \times\left(n_{0}+n_{1}+\ldots+n_{9}\right)$, where $n_{i}=$ numbers of plants at $i$ th scale of infection severity (11). An area under the disease progress curve (AUDPC) was calculated based on the severity on penultimate leaf according to the formula described by Bjarko and Line (2). Six wheat cultivars (Alidos, Andros, FAP77517, Massey, Mercia, and Redcoat) with known APR to powdery mildew were used as checks.

Statistical analysis. Relative AUDPC was calculated by dividing the AUDPC of each cultivar or line by the AUDPC of susceptible check Jingshuang 16. The percentage of maximum disease severity (MDS) on penultimate leaf was transformed into inverse sine by the formula $x=$ $\operatorname{Sin}^{-1} \sqrt{M D S}$ for subsequent analysis of variance (ANOVA) and protected least significant difference (LSD) test. SAS software (SAS Institute, Inc., Cary, NC) was used to compute ANOVA as well as the LSD test of differences between the relative AUDPC and MDS of each cultivar or line and the mean value of six check cultivars with known APR.

Table 1. Reaction patterns of 20 differential wheat cultivars and lines with known resistance genes to 20 isolates of Blumeria graminis f. sp. tritici collected from various regions of China

Infection types to $B$. graminis f. sp. tritici isolates ${ }^{a}$

\begin{tabular}{|c|c|c|c|c|c|c|c|c|c|c|c|c|c|c|c|c|c|c|c|c|c|}
\hline Cultivar or line & Gene $^{\text {b }}$ & E01 & E02 & E03 & E05 & E06 & E07 & E10 & E13 & E15 & E16 & E17 & E18 & E20 & E21 & E23 & E26 & E30 & E31 & E32 & $\overline{\mathbf{E 4 2}}$ \\
\hline Chancellor & None & 4 & 4 & 4 & 4 & 4 & 4 & 4 & 4 & 4 & 4 & 4 & 4 & 4 & 4 & 4 & 4 & 4 & 4 & 4 & 4 \\
\hline Axminster $/ 8 * \mathrm{CC}$ & Pml & 4 & 4 & 4 & 4 & 4 & 4 & 4 & 4 & 4 & 4 & 4 & 4 & 4 & 4 & 4 & 4 & 4 & 4 & 4 & 4 \\
\hline $\mathrm{Ulka} / 8 * \mathrm{CC}$ & Pm2 & 0 & 4 & 0 & 4 & 0 & 0 & 0 & 3 & 0 & 0 & 4 & 4 & 4 & 4 & 1 & 0 & 0 & 3 & 4 & 4 \\
\hline Maris Huntsman & $P m 2+6$ & 0 & 0 & 0 & 0 & 0 & 0 & 0 & 4 & 0 & - & 0 & 4 & 4 & 3 & 0 & 0 & 0 & 0 & 4 & 0 \\
\hline Maris Dove & $P m 2+M l d$ & 0 & 0 & 0 & 0 & 0 & 0 & 0 & 4 & 0 & 0 & 0 & 0 & 3 & 4 & 0 & 0 & 0 & 0 & 4 & 0 \\
\hline Brock & $P m 2+T a$ & 0 & 4 & 0 & 4 & 0 & 0 & 0 & 0 & 2 & 2 & 4 & 0 & 0 & 4 & 0 & 0 & 0 & 0 & 0 & 4 \\
\hline Asosan $/ 8 * \mathrm{CC}$ & $P m 3 a$ & 0 & 3 & 0 & 4 & 3 & 4 & 4 & 4 & 4 & 3 & 0 & 3 & 4 & 4 & 4 & 4 & 4 & 4 & 4 & 4 \\
\hline Kolibrí & $P m 3 d$ & 4 & 4 & 4 & 4 & 4 & 4 & 4 & 4 & 4 & 4 & 0 & 3 & 4 & 4 & 4 & 2 & 4 & 4 & 4 & 4 \\
\hline W150 & Pm3e & 4 & 4 & 4 & 4 & 4 & 4 & 4 & 4 & 4 & 4 & 4 & 4 & 4 & 4 & 4 & 4 & 4 & 4 & 4 & 4 \\
\hline $\begin{array}{l}\text { Michigen Amber/ } \\
8^{*} \mathrm{CC}\end{array}$ & $P m 3 f$ & 4 & 4 & 3 & 0 & 4 & 4 & 4 & 0 & 4 & 3 & 4 & 4 & 4 & 4 & 0 & 4 & 4 & 4 & 4 & 4 \\
\hline Khapli/8*CC & Pm4a & 0 & 4 & 0 & 0 & 1 & 0 & 4 & 0 & 4 & 4 & 4 & 4 & 4 & 4 & 1 & 0 & 1 & 0 & 0 & 0 \\
\hline Armada & $P m 4 b$ & 0 & 0 & 0 & 0 & 0 & 0 & 2 & 1 & 0 & 4 & 0 & 4 & 4 & 4 & 0 & 3 & 0 & 0 & 0 & 0 \\
\hline Baimian3 & $P m 4+8$ & 0 & 4 & 0 & 0 & 0 & 0 & 4 & 0 & 0 & 4 & 3 & 4 & 4 & 4 & 0 & 0 ; & 0 & 0 & 0 & 0 \\
\hline Hope & $P m 5$ & 4 & 4 & 4 & 4 & 4 & 4 & 4 & 4 & 4 & 4 & 4 & 4 & 4 & 4 & 4 & 4 & 4 & 4 & 4 & 4 \\
\hline Timgalen & Pm6 & 4 & 3 & 4 & 4 & 4 & 4 & 3 & 4 & 0 & 3 & 3 & 4 & 4 & 3 & 4 & 4 & 4 & 3 & 3 & 3 \\
\hline CI 14189 & $\operatorname{Pm} 7$ & 4 & 4 & 4 & 4 & 4 & 4 & 4 & 4 & 4 & 4 & 4 & 4 & 4 & 4 & 4 & 4 & 4 & 4 & 4 & 4 \\
\hline Kavkaz & Pm8 & 4 & 4 & 4 & 4 & 4 & 4 & 4 & 4 & 4 & 4 & 4 & 4 & 4 & 4 & 0 & 4 & 4 & 4 & 0 & 4 \\
\hline Wembley & Pm12 & 0 & 0 & 0 & 0 & 0 & 0 & 0 & 0 & 0 & 0 & 0 & 0 & 0 & 0 & 0 & 0 & 0 & 0 & 0 & 0 \\
\hline Brigrand & Pm16 & 0 & 0 & 0 & 0 & 1 & 0 & 0 & 0 & 0 & 0 & 0 & 0 & 2 & 0 & 0 & 0 & 0 & 0 & 0 & 0 \\
\hline Amigo & Pm17 & 4 & 4 & 4 & 4 & 3 & 4 & 3 & 4 & 0 & 4 & 0 & 4 & 4 & 4 & 0 & 4 & 4 & 3 & 0 & 4 \\
\hline $\operatorname{Pm} 20$ & Pm 20 & 0 & 0 & 0 & 0 & 0 & 0 & 0 & 3 & 0 & 0 & 0 & 0 & 2 & 4 & 0 & 2 & 0 & 0 & 0 & 0 \\
\hline
\end{tabular}

a Infection types are $0=$ no visible symptoms; $0 ;=$ hypersensitive necrotic flecks; 1 = minute colonies with few conidia; $2=$ colonies with moderately developed hyphae, but few conidia; 3 = colonies with well-developed hyphae and abundant conidia, but colonies not joined together; and $4=$ colonies with welldeveloped hyphae and abundant conidia, and colonies mostly joined together.

b Information obtained from Huang and Röder (16) and McIntosh et al. (24). 


\section{RESULTS}

Identification of resistance genes effective at the seedling stage. Cultivars and lines with resistance genes $P m l$, $P m 3 e, P m 5$, and $P m 7$ were susceptible to all isolates of $B$. graminis f. sp. tritici tested (Table 1). Genes Pm12 and Pm16 conferred resistance to all isolates, and Pm20 gave a susceptible reaction to isolates E13 and E21 only. Genes Pm2 and Pm6 conferred resistance to 10 and 1 isolates, respectively. Maris Huntsman, which carries both the Pm2 and Pm6 genes, was resistant to 14 isolates of $B$. graminis f. sp. tritici.

Of the 192 wheat cultivars and lines tested, 94 were susceptible to all isolates (Table 2). Ninety-eight wheat cultivars and lines were found to carry one or more re- sistance genes (Table 3). Of these, 11 cultivars and lines (Aiyuandong 3, CA8646, CA9632, Jinnong 8112, Lumai 13, Nongda 523, Zhoumai 13, Corydon, Jur/ 2*Iapar29//Pf83144, Kukuna, and Mv Optima) had the same reaction pattern as Kavkaz, which carries Pm8. Seven cultivars (BPM14, BPM28, CA9553, Lankao 906, Lu7you/C39, Nongdayoufan 5, and German 8661) showed the same reaction patterns as Armada, which carries $P m 4 b$. Aizao 4110 and CA9550 displayed a combination of reaction patterns of Ulka/ $8 * \mathrm{CC}$ and Armada, which carry $P m 2$ and $P m 4 b$, respectively. Three cultivars (90-I89, Shan 229, and Milan/Ducula) showed the same reaction pattern as Kolibri which carries $P m 3 d$, while seven cultivars or lines
(Zheng86115, 8030Versailles/Edch//Cd, Tnmu/Milan-2, Tx71A1039.V1*3/Ami// Trap\#1, Vee/Koel//He1/3*Cn079/3/Kauz, Soissons-2, and Soissons-3) showed evidence of unidentified $P m$ genes in addition to $P m 3 d$ (Table 3). Three cultivars (Nongda 280218, Nongda 883, and Rah122/94) showed the same resistance pattern as Khapli $/ 8^{*} \mathrm{CC}$, which carries Pm4a. BPM15 and Xingmai 883 displayed the same reaction patterns as Ulka/ $8 * \mathrm{CC}$ and Timgalen, respectively (Tables 2 and 3).

Twenty-one cultivars and lines (98301, Lumai 14, Shangdong200015537, Altar 84/Ae.squarrosa(219)//2*Seri, Br23/Emb40, Choix M95, Cty*3/Ta2450, Hahn/2* Weaver, Hussar, Irena/Weaver, Lfn/ Ii58.57//Prl/3/Hahn/4/2*Mo88, Mon/Tmu//

Table 2. Origin, growth habit, and 1B/1R translocation status of 94 cultivars and lines that were susceptible to 20 Blumeria graminis f. sp. tritici isolates

\begin{tabular}{|c|c|c|c|c|c|c|c|}
\hline Cultivar or line & Origin & $\begin{array}{c}\text { Growth } \\
\text { habit }^{\mathrm{a}}\end{array}$ & $\begin{array}{c}\text { 1B/1R } \\
\text { status }^{b}\end{array}$ & Cultivar or line & Origin & $\begin{array}{c}\text { Growth } \\
\text { habit }^{\mathrm{a}}\end{array}$ & $\begin{array}{c}\text { 1B/1R } \\
\text { status }^{b}\end{array}$ \\
\hline Bainong 64 & China & W & $1 \mathrm{~B} / 1 \mathrm{~B}$ & Bacanora T88 & CIMMYT & $\mathrm{S}$ & $1 \mathrm{~B} / 1 \mathrm{R}$ \\
\hline Baofeng 7228 & China & W & $1 \mathrm{~B} / 1 \mathrm{R}$ & Batera & CIMMYT & W & $\ldots$ \\
\hline CA 9532 & China & W & 1B/1R & Bow/Nkt//Ducula/3/Ducula & CIMMYT & $\mathrm{S}$ & $1 \mathrm{~B} / 1 \mathrm{~B}$ \\
\hline Hongjuanmang & China & W & 1B/1B & Emb27/Cep8825//Milan-1 & CIMMYT & S & $1 \mathrm{~B} / 1 \mathrm{~B}$ \\
\hline Hobbit/CA837 & China & $\mathrm{W}$ & $\ldots$ & Emb27/Cep8825//Milan-2 & CIMMYT & S & $1 \mathrm{~B} / 1 \mathrm{~B}$ \\
\hline Ji 966185 & China & $\mathrm{W}$ & $\ldots$ & F12.71/Bez//Tjb368.251/Buc/4/ & CIMMYT & W & $1 \mathrm{~B} / 1 \mathrm{~B}$ \\
\hline Jimai 30 & China & W & 1B/1R & D6301/Heine VII//ERA/3/BUC & & & \\
\hline Jimai 36 & China & W & 1B/1R & Filin & CIMMYT & $\mathrm{S}$ & $1 B / 1 R$ \\
\hline Jimai 38 & China & W & 1B/1R & Filin/Milan-1 & CIMMYT & S & $1 \mathrm{~B} / 1 \mathrm{~B}$ \\
\hline Jin 411 & China & W & 1B/1B & Filin/Milan-2 & CIMMYT & $S$ & $1 \mathrm{~B} / 1 \mathrm{~B}$ \\
\hline Jinan 17 & China & W & 1B/1B & Gen/Kauz & CIMMYT & S & $1 B / 1 R$ \\
\hline Jingdong 6 & China & W & 1B/1R & Hxl 8088/Ducula & CIMMYT & S & $\ldots$ \\
\hline Jingdong 8 & China & W & 1B/1R & Inqalab 91 & CIMMYT & $S$ & $1 \mathrm{~B} / 1 \mathrm{~B}$ \\
\hline Jinmai 45 & China & W & 1B/1R & Milan & CIMMYT & S & $1 \mathrm{~B} / 1 \mathrm{~B}$ \\
\hline Jinmai 47 & China & W & $\ldots$ & Milan/Pastor & CIMMYT & $S$ & $1 \mathrm{~B} / 1 \mathrm{~B}$ \\
\hline Linfen 6010 & China & W & $\ldots$ & Saar & CIMMYT & $S$ & $1 \mathrm{~B} / 1 \mathrm{~B}$ \\
\hline Lumai 21 & China & W & 1B/1B & Sha3/Seri//Sha4/Lira & CIMMYT & S & $1 B / 1 R$ \\
\hline Lumai 22 & China & $\mathrm{W}$ & 1B/1B & Thb//Maya/Nac/3/Rabe/4/Milan & CIMMYT & S & $1 \mathrm{~B} / 1 \mathrm{~B}$ \\
\hline Lumai 23 & China & W & 1B/1B & Ures/Kauz & CIMMYT & S & $1 B / 1 R$ \\
\hline Lunhui 201 & China & W & $1 \mathrm{~B} / 1 \mathrm{R}$ & Dulus & CIMMYT & $S$ & $1 \mathrm{~B} / 1 \mathrm{~B}$ \\
\hline Nongda123 & China & W & 1B/1B & Heilo & CIMMYT & $S$ & $1 \mathrm{~B} / 1 \mathrm{~B}$ \\
\hline Pm 20491 & China & W & 1B/1B & Prointa Guazu & Argentina & $S$ & $1 B / 1 R$ \\
\hline Pm 499-1 & China & W & 1B/1B & Prointa Cauquen & Argentina & $\mathrm{S}$ & $1 \mathrm{~B} / 1 \mathrm{~B}$ \\
\hline Pm94081 & China & W & $\ldots$ & $110254-1.77 \mathrm{~W}$ & France & W & $\ldots$ \\
\hline Pm94082 & China & W & $\ldots$ & Cp9106-1-2-1 & France & W & $\ldots$ \\
\hline Shan 213 & China & W & 1B/1R & Cp9111-3-2 & France & W & $\ldots$ \\
\hline Shan 354 & China & W & 1B/1R & Pistov-2 & France & W & $\ldots$ \\
\hline Shangdong 935031 & China & W & 1B/1B & Alidos & Germany & W & $\ldots$ \\
\hline Shannong413863 & China & W & 1B/1R & Andros & Germany & W & $1 \mathrm{~B} / 1 \mathrm{~B}$ \\
\hline Tiegangmai & China & W & 1B/1B & Mv Emma & Hungary & $\mathrm{W}$ & $1 \mathrm{~B} / 1 \mathrm{R} *$ \\
\hline Wenmai 6 & China & W & 1B/1B & Mv Magdalena & Hungary & W & 1B/1R \\
\hline Xiaoyan 6 & China & W & 1B/1B & Mv Magvas & Hungary & W & $1 \mathrm{~B} / 1 \mathrm{~B}$ \\
\hline Xingmai 99 & China & W & 1B/1R & Mv Palma & Hungary & W & $1 \mathrm{~B} / 1 \mathrm{R} *$ \\
\hline Yumai 13 & China & W & 1B/1R & Mv Vilma & Hungary & W & $\ldots$ \\
\hline Yumai 18 & China & W & 1B/1B & Dropia & Romania & W & $\ldots$ \\
\hline Yumai 21 & China & W & 1B/1R & Arina & Switzerland & $\mathrm{W}$ & $1 \mathrm{~B} / 1 \mathrm{~B}$ \\
\hline Yumai 25 & China & $\mathrm{W}$ & 1B/1B & Kariega & South Africa & $\mathrm{S}$ & $1 \mathrm{~B} / 1 \mathrm{~B}$ \\
\hline Yumai 47 & China & W & 1B/1B & Manning/SDV1//DOGU88 & Turkey & W & $1 \mathrm{~B} / 1 \mathrm{~B}$ \\
\hline Yuzhan 9705 & China & W & $\ldots$ & Abn/Jun & United States & W & $1 \mathrm{~B} / 1 \mathrm{~B}$ \\
\hline Zhengmai 9405 & China & W & 1B/1B & Ga841465-2-1-1-4 & United States & $\mathrm{W}$ & $\ldots$ \\
\hline Zhongyou 9507 & China & W & 1B/1B & Ga85164-3-2-4-2 & United States & $\mathrm{W}$ & $\ldots$ \\
\hline Zhongyou 9701 & China & $\mathrm{W}$ & 1B/1B & Houser & United States & W & $1 \mathrm{~B} / 1 \mathrm{~B}$ \\
\hline Zhongyou 9843 & China & $\mathrm{W}$ & 1B/1B & Ks90175-1-2 & United States & $\mathrm{W}$ & $1 B / 1 R$ \\
\hline Zhongyou 9844 & China & W & 1B/1B & Redcoat & United States & W & $1 \mathrm{~B} / 1 \mathrm{~B}$ \\
\hline Zhoumai 11 & China & $\mathrm{W}$ & 1B/1R & Mercia & United Kingdom & $\mathrm{W}$ & $1 \mathrm{~B} / 1 \mathrm{~B}$ \\
\hline Zhoumai 16 & China & $\mathrm{W}$ & 1B/1R & FAP74161 & $\cdots$ & $\mathrm{W}$ & $1 \mathrm{~B} / 1 \mathrm{~B}$ \\
\hline Zhoumai 17 & China & W & 1B/1R & FAP77517 & $\ldots$ & W & $1 \mathrm{~B} / 1 \mathrm{R}$ \\
\hline 24Ibwsn\#278/Soissons & CIMMYT & $\mathrm{W}$ & 1B/1B & & & & \\
\hline
\end{tabular}

a $\mathrm{W}=$ winter wheat; $\mathrm{S}=$ spring wheat.

${ }^{\mathrm{b}}$ Identified according to the method described by Gupta and Shepherd (12), except for those indicated by an asterisk, for which information obtained from Schlegel (27); ... = unknown. 
Ald/Pvn, Mv Madrigal, Mv Magdalena, Nd610/Kauz//Tui, Prinia, Tinamou, Tnmu/ Milan-1, Tnmu/Milan-3, Tnmu/Milan-4, and $\mathrm{Vee} / \mathrm{Koel} / / \mathrm{He} 1 / 3 * \mathrm{Cn} 079 / 3 / \mathrm{Kauz})$ showed evidence of unidentified resistance genes in addition to $P m 8$.

Thirty-six cultivars were resistant to one or several isolates; however, their reaction patterns did not match any of the known resistance genes or their combinations. Of these, Mv-17, Va91-51-26, Va91-52-65, and Pm93-625-4 were resistant to all isolates and showed the same reaction pattern as those of Pm12 and Pml6.
APR to powdery mildew. Cultivars and lines differed significantly for relative AUDPC and MDS in the field trials (Table 4). Twenty-two cultivars and lines showed APR to powdery mildew (Table $5)$. The disease developed quickly on the susceptible check, Jingshuang 16, indicating a favorable environment for disease development (Fig. 1). Compared with susceptible cultivars, disease developed much more slowly on APR cultivars (Fig. 1).

Among the cultivars and lines, 11 (including 5 APR checks) were susceptible to all isolates of $B$. graminis f. sp. tritici at the seedling stage, but showed slow disease progress at the adult stage in the field. In all, 17 cultivars (including 1 APR check) with resistance genes overcome by isolate E20 at the seedling stage showed moderate resistance in the field (Tables 3 and 5). In contrast, Aizao 4110, which carries $P m 2+4 b$, was highly susceptible in the field, with a relative AUDPC and MDS of 0.73 and 42.7, respectively. A highly significant correlation was found between AUDPC and MDS on penultimate leaf $(r=0.96, P<0.001)$.

Table 3. Reactions of 98 wheat cultivars or lines to 20 isolates of Blumeria graminis f. sp. tritici in seedling tests

\begin{tabular}{|c|c|c|c|c|c|c|c|c|c|c|c|c|c|c|c|c|c|c|c|c|c|c|c|c|}
\hline \multirow[b]{2}{*}{ Cultivar or line } & \multirow[b]{2}{*}{ Origin } & \multirow[b]{2}{*}{$\mathbf{G H}^{\mathbf{b}}$} & \multirow[b]{2}{*}{ Status ${ }^{c}$} & \multirow[b]{2}{*}{ Gene } & \multicolumn{20}{|c|}{ Infection types to $B$. graminis f. sp. tritici isolates ${ }^{a}$} \\
\hline & & & & & $\begin{array}{l}\mathbf{E} \\
\mathbf{0 1}\end{array}$ & $\begin{array}{c}\mathbf{E} \\
\mathbf{0 2}\end{array}$ & $\begin{array}{c}\mathbf{E} \\
\mathbf{0 3}\end{array}$ & $\begin{array}{c}\mathbf{E} \\
\mathbf{0 5}\end{array}$ & $\begin{array}{c}\mathbf{E} \\
06\end{array}$ & $\begin{array}{c}\mathbf{E} \\
\mathbf{0 7}\end{array}$ & $\begin{array}{c}\mathbf{E} \\
10\end{array}$ & $\begin{array}{c}E \\
13\end{array}$ & $\begin{array}{c}\mathbf{E} \\
15\end{array}$ & $\begin{array}{c}E \\
16\end{array}$ & $\begin{array}{c}\mathbf{E} \\
17\end{array}$ & $\begin{array}{c}E \\
18\end{array}$ & $\begin{array}{c}\mathbf{E} \\
20\end{array}$ & $\begin{array}{c}E \\
21\end{array}$ & $\begin{array}{c}E \\
23\end{array}$ & $\begin{array}{c}E \\
26\end{array}$ & $\begin{array}{c}\mathbf{E} \\
\mathbf{3 0}\end{array}$ & $\begin{array}{c}\mathbf{E} \\
\mathbf{3 1}\end{array}$ & $\begin{array}{c}\mathbf{E} \\
\mathbf{3 2}\end{array}$ & $\begin{array}{c}E \\
42\end{array}$ \\
\hline 98301 & China & W & $1 \mathrm{~B} / 1 \mathrm{R}$ & Pm8+? & 4 & 4 & 4 & 4 & 4 & 4 & 4 & 4 & 4 & 4 & 4 & 4 & 4 & 3 & 1 & 4 & 0 & 4 & 0 & 4 \\
\hline 975297 & China & $\mathrm{W}$ & $1 \mathrm{~B} / 1 \mathrm{R}$ & $?$ & 4 & 4 & 4 & 4 & 4 & 4 & 4 & 4 & 4 & 4 & 4 & 4 & 4 & 4 & 3 & 4 & 4 & 4 & 0 & 4 \\
\hline 98Zhong 33 & China & W & $1 \mathrm{~B} / 1 \mathrm{~B}$ & $?$ & 0 & 0 & 4 & 4 & 0 & 0 & 4 & 0 & 0 & 4 & 0 & 4 & 4 & 4 & 0 & 0 & 0 & 0 & 0 & 4 \\
\hline Aiyuandong 3 & China & W & $1 \mathrm{~B} / 1 \mathrm{R}$ & $\operatorname{Pm} 8$ & 4 & 4 & 4 & 4 & 4 & 4 & 4 & 4 & 4 & 4 & 4 & 4 & 4 & 4 & 0 & 4 & 3 & 4 & 0 & 4 \\
\hline Aizao 4110 & China & W & $1 \mathrm{~B} / 1 \mathrm{~B}$ & $P m 2+4 b$ & 0 & 0 & 0 & 0 & 0 & 0 & 0 & 1 & 0 & 2 & 0 & 4 & 4 & 4 & 0 & 0 & 0 & 0 & 0 & 0 \\
\hline BPM 14 & China & W & $1 \mathrm{~B} / 1 \mathrm{~B}$ & $P m 4 b$ & 0 & 0 & 0 & 0 & 2 & 0 & 0 & 0 & 0 & 4 & 0 & 4 & 3 & 4 & 0 & 4 & 2 & 0 & 0 & 0 \\
\hline BPM 15 & China & $\mathrm{W}$ & $1 \mathrm{~B} / 1 \mathrm{~B}$ & $P m 2$ & 0 & 4 & 0 & 4 & 0 & 0 & 1 & 3 & 0 & 0 & 4 & 4 & 4 & 4 & 0 & 0 & 0 & 3 & 4 & 4 \\
\hline BPM 28 & China & W & $1 \mathrm{~B} / 1 \mathrm{~B}$ & $P m 4 b$ & 0 & 0 & 0 & 0 & 2 & 0 & 0 & 0 & 0 & 4 & 0 & 3 & 4 & 4 & 1 & 4 & 2 & 0 & 0 & 0 \\
\hline CA 8646 & China & $\mathrm{W}$ & $1 \mathrm{~B} / 1 \mathrm{R}$ & $\operatorname{Pm} 8$ & 4 & 4 & 4 & 4 & 4 & 4 & 4 & 4 & 4 & 4 & 4 & 4 & 4 & 4 & 0 & 4 & 3 & 4 & 0 & 4 \\
\hline CA 9550 & China & W & $1 \mathrm{~B} / 1 \mathrm{~B}$ & $P m 2+4 b$ & 0 & 0 & 0 & 0 & 0 & 0 & 0 & 0 & 0 & 0 & 0 & 4 & 4 & 4 & 0 & 0 & 0 & 0 & 0 & 0 \\
\hline CA 9553 & China & $\mathrm{W}$ & 1B/1B & $P m 4 b$ & 0 & 0 & 0 & 0 & 0 & 0 & 1 & 1 & 0 & 3 & 0 & 4 & 3 & 4 & 0 & 4 & 0 & 0 & 0 & 0 \\
\hline CA 9632 & China & W & $1 \mathrm{~B} / 1 \mathrm{R}$ & Pm8 & 4 & 4 & 4 & 4 & 4 & 4 & 4 & 4 & 4 & 4 & 4 & 4 & 4 & 4 & 1 & 4 & 3 & 4 & 0 & 4 \\
\hline CA 9641 & China & W & $1 \mathrm{~B} / 1 \mathrm{~B}$ & $P m 3 a+?$ & 0 & 0 & 0 & 0 & 4 & 0 & 0 & 4 & 0 & 0 & 0 & 4 & 4 & 4 & 0 & 1 & 0 & 0 & 4 & 0 \\
\hline CA 8686 & China & W & $1 \mathrm{~B} / 1 \mathrm{R}$ & $?$ & 4 & 3 & 4 & 4 & 4 & 4 & 4 & 4 & 4 & 4 & 4 & 4 & 4 & 4 & 2 & 4 & 4 & 4 & 4 & 4 \\
\hline CA 9722 & China & $\mathrm{W}$ & $1 \mathrm{~B} / 1 \mathrm{~B}$ & $?$ & 0 & 4 & 0 & 0 & 3 & 0 & 4 & 4 & 4 & 4 & 4 & 4 & 4 & 4 & 4 & 3 & 2 & 0 & 0 & 0 \\
\hline Jimai 3 & China & W & $1 \mathrm{~B} / 1 \mathrm{~B}$ & $?$ & 4 & 4 & 4 & 4 & 4 & 0 & 4 & 4 & 4 & 4 & 4 & 4 & 4 & 4 & 4 & 4 & 0 & 4 & 0 & 4 \\
\hline Jinnong 8112 & China & $\mathrm{W}$ & $1 \mathrm{~B} / 1 \mathrm{R}$ & $\operatorname{Pm} 8$ & 4 & 4 & 4 & 4 & 4 & 4 & 4 & 4 & 4 & 4 & 4 & 4 & 4 & 4 & 1 & 4 & 3 & 4 & 0 & 4 \\
\hline Lankao 906 & China & $\mathrm{W}$ & $1 \mathrm{~B} / 1 \mathrm{~B}$ & $P m 4 b$ & 0 & 0 & 0 & 0 & 2 & 0 & 0 & 0 & 0 & 4 & 0 & 4 & 4 & 4 & 0 & 4 & 2 & 0 & 0 & 0 \\
\hline Lin 9303 & China & $\mathrm{W}$ & $1 \mathrm{~B} / 1 \mathrm{R}$ & $?$ & 4 & 4 & 4 & 4 & 4 & 4 & 4 & 4 & 4 & 4 & 4 & 4 & 4 & 4 & 4 & 4 & 2 & 4 & 0 & 4 \\
\hline Lu7you/2/C39 & China & W & $1 \mathrm{~B} / 1 \mathrm{~B}$ & $\mathrm{Pm} 4 b$ & 0 & 0 & 0 & 2 & 0 & 0 & 0 & 0 & 0 & 3 & 0 & 4 & 4 & 4 & 0 & 3 & 0 & 0 & 0 & 0 \\
\hline Lumai 13 & China & W & $1 \mathrm{~B} / 1 \mathrm{R}$ & $\operatorname{Pm} 8$ & 4 & 4 & 4 & 4 & 4 & 4 & 4 & 4 & 4 & 4 & 4 & 4 & 4 & 4 & 1 & 4 & 3 & 4 & 0 & 4 \\
\hline Lumai 14 & China & $\mathrm{W}$ & $1 \mathrm{~B} / 1 \mathrm{R}$ & Pm8+? & 4 & 4 & 4 & 4 & 4 & 4 & 4 & 4 & 4 & 4 & 4 & 4 & 4 & 4 & 1 & 4 & 0 & 4 & 0 & 4 \\
\hline Neixiang 991 & China & $\mathrm{W}$ & $1 \mathrm{~B} / 1 \mathrm{R}$ & $P m 2+6+?$ & - & 0 & 0 & 0 & 0 & 0 & 0 & 0 & 0 & 4 & 0 & 0 & 4 & 4 & 4 & 0 & 0 & 0 & 0 & 0 \\
\hline Neixiang 188 & China & $\mathrm{W}$ & $1 B / 1 R$ & $?$ & 4 & 4 & 4 & 4 & 4 & 4 & 4 & 4 & 4 & 0 & 4 & 4 & 4 & 4 & 4 & 4 & 4 & 4 & 4 & 4 \\
\hline Nongda 280218 & China & $\mathrm{W}$ & $1 \mathrm{~B} / 1 \mathrm{~B}$ & $P m 4 a$ & 0 & 4 & 0 & 0 & 1 & 0 & 3 & 0 & 4 & 4 & 3 & 3 & 4 & 4 & 0 & 0 & 1 & 1 & 0 & 0 \\
\hline Nongda 523 & China & W & $1 \mathrm{~B} / 1 \mathrm{R}$ & Pm8 & 4 & 4 & 4 & 4 & 4 & 4 & 4 & 4 & 4 & 4 & 4 & 4 & 4 & 4 & 0 & 4 & 3 & 4 & 0 & 4 \\
\hline Nongda 883 & China & W & $\ldots$ & $P m 4 a$ & 0 & 4 & 0 & 0 & 2 & 0 & 3 & 0 & 4 & 4 & 4 & 4 & 3 & 4 & 0 & 0 & 1 & 2 & 0 & 0 \\
\hline Nongdayoufan 5 & China & W & $\ldots$ & $P m 4 b$ & 0 & 0 & 0 & 2 & 0 & 0 & 0 & 2 & 0 & 3 & 0 & 4 & 4 & 4 & 0 & 3 & 0 & 0 & 0 & 0 \\
\hline Qiandong 6 & China & W & $\ldots$ & $P m 2+T a$ & 2 & 4 & 0 & 4 & 0 & 0 & 2 & 0 & 0 & 2 & 4 & 0 & 0 & 4 & 0 & 0 & 2 & 0 & 0 & 4 \\
\hline Pm93-625-4 & China & $\mathrm{W}$ & $1 \mathrm{~B} / 1 \mathrm{~B}$ & $\operatorname{Pm} 30$ & 0 & 0 & 0 & 0 & 0 & 0 & 0 & 0 & 0 & 0 & 0 & 0 & 0 & 0 & 0 & 0 & 0 & 0 & 0 & 0 \\
\hline Shan 229 & China & $\mathrm{W}$ & $1 \mathrm{~B} / 1 \mathrm{R}$ & $\operatorname{Pm} 3 d$ & 4 & 4 & 4 & 4 & 4 & 4 & 4 & 4 & 4 & 4 & 0 & 3 & 4 & 4 & 4 & 0 & 4 & 4 & 4 & 4 \\
\hline Shangdong 20015537 & China & $\mathrm{W}$ & $1 \mathrm{~B} / 1 \mathrm{R}$ & Pm8+? & 4 & 4 & 4 & 4 & 4 & 4 & 4 & 4 & 4 & 4 & 4 & 4 & 4 & 4 & 1 & 4 & 0 & 4 & 0 & 4 \\
\hline Shangdong 418 & China & $\mathrm{W}$ & $1 \mathrm{~B} / 1 \mathrm{R}$ & $?$ & 4 & 4 & 0 & 4 & 4 & 4 & 4 & 4 & 4 & 3 & 4 & 4 & 4 & 4 & 4 & 4 & 4 & 4 & 4 & 4 \\
\hline Xingmai 883 & China & $\mathrm{W}$ & $\ldots$ & Pm6 & 4 & 4 & 4 & 4 & 4 & 4 & 4 & 4 & 0 & 4 & 4 & 4 & 4 & 4 & 4 & 4 & 4 & 4 & 4 & 4 \\
\hline YE-2416-7 ${ }^{\mathrm{a}}$ & China & W & $1 \mathrm{~B} / 1 \mathrm{~B}$ & $?$ & 4 & 0 & 4 & 4 & 4 & 3 & 4 & 4 & 0 & 4 & 0 & & 4 & 4 & 0 & 3 & 1 & 0 & 0 & 0 \\
\hline Zheng 86115 & China & $\mathrm{W}$ & $1 \mathrm{~B} / 1 \mathrm{~B}$ & $P m 3 d+?$ & 4 & 3 & 0 & 3 & 4 & 4 & 4 & 4 & 4 & 4 & 2 & 3 & 4 & 4 & 4 & 3 & 4 & 4 & 4 & 4 \\
\hline Zhoumai 13 & China & $\mathrm{W}$ & $1 \mathrm{~B} / 1 \mathrm{R}$ & $\operatorname{Pm} 8$ & 4 & 4 & 4 & 4 & 4 & 4 & 4 & 4 & 4 & 4 & 4 & 4 & 4 & 4 & 0 & 4 & 3 & 4 & 0 & 4 \\
\hline Zhoumai 88 & China & $\mathrm{W}$ & $1 \mathrm{~B} / 1 \mathrm{~B}$ & $?$ & 4 & 4 & 4 & 4 & 4 & 4 & 4 & 4 & 4 & 4 & 4 & 4 & 4 & 4 & 4 & 4 & 3 & 4 & 0 & 4 \\
\hline 494J6.11 & CIMMYT & $\mathrm{W}$ & $1 \mathrm{~B} / 1 \mathrm{~B}$ & $?$ & 4 & 0 & 4 & 4 & 4 & 4 & 4 & 4 & 4 & 4 & 4 & 4 & 4 & 4 & 4 & 4 & 4 & 0 & 4 & 4 \\
\hline 8030 Versailles/Edch//Cd & CIMMYT & W & $1 \mathrm{~B} / 1 \mathrm{R}$ & $P m 3 d+?$ & 4 & 0 & 4 & 4 & 3 & 2 & 4 & 4 & 4 & 4 & 0 & 3 & 4 & 4 & 4 & 2 & 4 & 3 & 4 & 4 \\
\hline $\begin{array}{l}\text { Altar 84/Ae.squarrosa } \\
(219) / / 2 * \text { Seri }\end{array}$ & CIMMYT & $\mathrm{S}$ & $1 \mathrm{~B} / 1 \mathrm{R}$ & $P m 8+?$ & 4 & 0 & 0 & 4 & 4 & 4 & 4 & 4 & 0 & 4 & 0 & 0 & 0 & 4 & 0 & 4 & 4 & 0 & 0 & 4 \\
\hline $\begin{array}{l}\mathrm{Au} / \mathrm{Up} 301 / / \mathrm{Gll} / \mathrm{Sx} / 3 / \mathrm{Pew} / 4 / \\
\mathrm{Mai} / \mathrm{Maya} / / \mathrm{Pew} / 5 / \mathrm{Kea} / 6 / 2 * \mathrm{R} 37 / \\
\mathrm{Gh} 1121 / / \mathrm{Kal} / \mathrm{Bb} / 3 / \mathrm{Buc} / \mathrm{Bul}\end{array}$ & CIMMYT & $\mathrm{S}$ & $1 \mathrm{~B} / 1 \mathrm{~B}$ & $?$ & 4 & 0 & 4 & 4 & 4 & 4 & 4 & 4 & 4 & 4 & 4 & 4 & 4 & 4 & 4 & 4 & 4 & 4 & 4 & 4 \\
\hline Catbird & CIMMYT & $\mathrm{S}$ & $1 \mathrm{~B} / 1 \mathrm{R}$ & $?$ & 4 & 0 & 4 & 4 & 4 & 4 & 4 & 4 & 4 & 4 & 4 & 4 & 4 & 4 & 4 & 4 & 4 & 3 & 4 & 4 \\
\hline Croc_1/Ae.squarrosa(205) //Kauz & CIMMYT & $\mathrm{S}$ & $1 \mathrm{~B} / 1 \mathrm{R}$ & $?$ & 4 & 4 & 4 & 4 & 2 & 4 & 4 & 4 & 4 & 4 & 4 & 4 & 4 & 4 & 4 & 4 & 4 & 4 & 4 & 4 \\
\hline Choix M95 & CIMMYT & $\mathrm{S}$ & $1 \mathrm{~B} / 1 \mathrm{R}$ & $P m 8+?$ & 4 & 4 & 4 & 4 & 4 & 4 & 4 & 4 & 4 & 4 & 4 & 4 & 4 & 4 & $\begin{array}{l}1 \\
(\mathrm{col})\end{array}$ & $\begin{array}{c}4 \\
\text { ntinu }\end{array}$ & $\begin{array}{l}0 \\
\text { ledo }\end{array}$ & $\begin{array}{c}4 \\
n \text { ne }\end{array}$ & $\begin{array}{l}0 \\
x t p c\end{array}$ & $\begin{array}{c}4 \\
\text { age })\end{array}$ \\
\hline
\end{tabular}




\section{DISCUSSION}

In the present study, known powdery mildew resistance genes were detected in 62 wheat cultivars and lines. Of these resistance genes, $P m 12$ and $P m 16$ were resistant to all isolates of $B$. graminis $\mathrm{f}$. sp. tritici tested, indicating that they are effective resistance genes against powdery mildew in China. Pm20 was resistant to most of the isolates and would confer effective resistance, at least in areas where virulence may be absent. Nevertheless, it is essential to monitor the virulence isolates of $B$. graminis f. sp. tritici to these resistance genes and diversify the genes in wheat cultivars. Genes $P m 2$ and Pm6 conferred resistance to 10 and 1 isolates, respectively. However, Maris Huntsman, which carries both the Pm2 and Pm6 genes, was resistant to 14 isolates of $B$. graminis f. sp. tritici, indicating the presence of a previously unidentified resistance gene or genes in this cultivar. The resistance gene in the line Pm93-625-4 was derived from wild emmer (accession C20), which should be Pm30 according to its origin.

Pm8 was the most frequently identified resistance gene, followed by $P m 4 b$ in Chinese wheat cultivars. $P m 8$ in these culti- vars is derived mainly from the $1 \mathrm{~B} / 1 \mathrm{R}$ cvs. Lovrin 13, Predgornaia 2, Kavkaz, Neuzucht, and their derivatives, which have been widely employed in wheat breeding programs in China since the early 1970s (13). At present, $46 \%$ of Chinese wheat cultivars possess the $1 \mathrm{~B} / 1 \mathrm{R}$ translocation (Z. L. Wang, unpublished). The high frequency of virulence to $P m 8$ in Chinese $B$. graminis f. sp. tritici populations has remained stable at about $94 \%$ (6). This has led to increased losses in China due to powdery mildew.

All Chinese wheat cultivars and lines carrying the $P m 8$ gene possess the $1 \mathrm{~B} / 1 \mathrm{R}$

Table 3. (continued from preceding page)

Infection types to $B$. graminis f. sp. tritici isolates ${ }^{\text {a }}$

\begin{tabular}{|c|c|c|c|c|c|c|c|c|c|c|c|c|c|c|c|c|c|c|c|c|c|c|c|c|}
\hline Cultivar or line & Origin & $\mathbf{G H}^{\mathbf{b}}$ & Status ${ }^{c}$ & Gene & $\begin{array}{l}\mathbf{E} \\
\mathbf{0 1}\end{array}$ & $\begin{array}{l}\mathbf{E} \\
\mathbf{0 2}\end{array}$ & $\begin{array}{c}\mathbf{E} \\
\mathbf{0 3}\end{array}$ & $\begin{array}{c}\mathbf{E} \\
\mathbf{0 5}\end{array}$ & $\begin{array}{l}\mathbf{E} \\
06\end{array}$ & $\begin{array}{l}\mathbf{E} \\
\mathbf{0 7}\end{array}$ & $\begin{array}{c}\mathbf{E} \\
\mathbf{1 0}\end{array}$ & $\begin{array}{c}E \\
13\end{array}$ & $\begin{array}{c}\mathbf{E} \\
15\end{array}$ & $\begin{array}{l}E \\
16\end{array}$ & 17 & 18 & $\begin{array}{c}\mathbf{E} \\
\mathbf{2 0}\end{array}$ & 21 & $\begin{array}{c}\mathbf{E} \\
23\end{array}$ & 26 & $\begin{array}{c}\mathbf{E} \\
\mathbf{3 0}\end{array}$ & $\begin{array}{c}\mathbf{E} \\
\mathbf{3 1}\end{array}$ & $\begin{array}{c}\mathbf{E} \\
\mathbf{3 2}\end{array}$ & $\begin{array}{r}E \\
42\end{array}$ \\
\hline Corydon & CIMMYT & $\mathrm{S}$ & $1 \mathrm{~B} / 1 \mathrm{R}$ & $\operatorname{Pm} 8$ & 4 & 4 & 4 & 4 & 4 & 4 & 4 & 4 & 4 & 4 & 4 & 4 & 4 & 4 & 1 & 4 & 3 & 4 & 0 & + \\
\hline Desconocido & CIMMYT & $\mathrm{S}$ & $1 \mathrm{~B} / 1 \mathrm{R}$ & $?$ & 4 & 4 & 0 & 4 & 4 & 4 & 4 & 4 & 4 & 4 & 4 & 4 & 4 & 4 & 4 & 4 & 4 & 4 & 4 & 4 \\
\hline Ducula & CIMMYT & $\mathrm{S}$ & $1 \mathrm{~B} / 1 \mathrm{~B}$ & $?$ & 4 & 4 & 4 & 4 & 4 & 4 & 4 & 4 & 4 & 4 & 4 & 0 & 4 & 4 & 4 & 4 & 4 & 4 & 4 & \\
\hline Hahn/2*Weaver & CIMMYT & $\mathrm{S}$ & $1 \mathrm{~B} / 1 \mathrm{R}$ & Pm8+? & 4 & 4 & 4 & 4 & 4 & 4 & 4 & 4 & 4 & 4 & 4 & 4 & 4 & 4 & 0 & 4 & 0 & 4 & 0 & 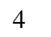 \\
\hline Irena/Weaver & CIMMYT & $\mathrm{S}$ & $1 \mathrm{~B} / 1 \mathrm{R}$ & Pm8+? & 4 & 4 & 4 & 4 & 4 & 4 & 4 & 4 & 4 & 4 & 4 & 4 & 4 & 4 & 2 & 4 & 0 & 4 & 0 ; & \\
\hline Ketupa & CIMMYT & $\mathrm{S}$ & $1 \mathrm{~B} / 1 \mathrm{R}$ & $?$ & 4 & 0 & 0 & 4 & 4 & 4 & 4 & 4 & 0 & 4 & 0 & 4 & 4 & 3 & 4 & 4 & 4 & 0 & 4 & 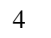 \\
\hline Kukuna & CIMMYT & $\mathrm{S}$ & $1 \mathrm{~B} / 1 \mathrm{R}$ & $\operatorname{Pm} 8$ & 4 & 3 & 4 & 4 & 4 & 4 & 4 & 4 & 4 & 4 & 4 & 4 & 4 & 4 & 1 & 4 & 3 & 4 & 0 & \\
\hline $\begin{array}{l}\text { Lfn/Ii58.57//Prl/3/Hahn/4/ } \\
\text { 2*Mo88 }\end{array}$ & CIMMYT & $\mathrm{S}$ & $1 B / 1 R$ & Pm8+? & 4 & 4 & 4 & 4 & 4 & 4 & 4 & 4 & 4 & 4 & 4 & 4 & 4 & 4 & 0 & 4 & 0 & 4 & 0 & \\
\hline Milan/Ducula & CIMMYT & $\mathrm{S}$ & $1 \mathrm{~B} / 1 \mathrm{~B}$ & $P m 3 d$ & 4 & 4 & 4 & 4 & 4 & 4 & 4 & 4 & 4 & 4 & 0 & 4 & 4 & 4 & 4 & 2 & 4 & 4 & 4 & 4 \\
\hline Mon/Tmu//Ald/Pvn & CIMMYT & $\mathrm{S}$ & $1 \mathrm{~B} / 1 \mathrm{R}$ & Pm8+? & 4 & 4 & 4 & 4 & 4 & 4 & 4 & 4 & 4 & 4 & 4 & 4 & 4 & 4 & 1 & 4 & 0 & 4 & 0 & \\
\hline Nadadores M63 & CIMMYT & $\mathrm{S}$ & $1 \mathrm{~B} / 1 \mathrm{~B}$ & $?$ & 4 & 4 & 0 & 4 & 4 & 4 & 4 & 4 & 4 & 4 & 4 & 0 & 4 & 4 & 4 & 4 & 4 & 4 & 0 & \\
\hline Otus & CIMMYT & $\mathrm{S}$ & $1 \mathrm{~B} / 1 \mathrm{R}$ & $?$ & 4 & 3 & 4 & 4 & 4 & 4 & 4 & 4 & 4 & 4 & 4 & 4 & 4 & 4 & 0 & 4 & 4 & 4 & 4 & \\
\hline Pastor & CIMMYT & $\mathrm{S}$ & $1 \mathrm{~B} / 1 \mathrm{~B}$ & $?$ & 4 & 0 & 2 & 4 & 4 & 4 & 4 & 4 & 4 & 4 & 4 & 4 & 4 & 4 & 4 & 4 & 3 & 3 & 4 & 4 \\
\hline Pbw343 & CIMMYT & $\mathrm{S}$ & $1 B / 1 R$ & $?$ & 4 & 0 & 0 & 4 & 4 & 4 & 4 & 4 & 4 & 4 & 4 & 4 & 4 & 4 & 4 & 4 & 4 & 0 & 4 &  \\
\hline Prinia & CIMMYT & $\mathrm{S}$ & $1 B / 1 R$ & Pm8+6+? & 4 & 0 & 0 & 4 & 4 & 4 & 4 & 4 & 0 & 4 & 3 & 0 & 3 & 4 & 0 & 4 & 0 & 0 & 0 & 4 \\
\hline Seri/Attila & CIMMYT & S & $1 \mathrm{~B} / 1 \mathrm{R}$ & $?$ & 4 & 0 & 0 & 4 & 4 & 4 & 4 & 4 & 4 & 4 & 4 & 4 & 4 & 4 & 4 & 4 & 4 & 3 & 4 & \\
\hline Seri/Rayon & CIMMYT & S & $1 \mathrm{~B} / 1 \mathrm{~B}$ & $P m 3 d+?$ & 4 & 0 & 0 & 4 & 4 & 4 & 4 & 4 & 4 & 4 & 0 & 0 & 4 & 4 & 4 & 1 & 4 & 3 & 4 & 4 \\
\hline Tinar & CIMMYT & S & $1 B / 1 R$ & Pm8+6+? & 4 & 0 & 0 & 4 & 4 & 4 & 4 & 4 & 0 & 4 & 4 & 4 & 0 & 4 & 0 & 4 & 0 & 0 & 0 & \\
\hline Tnmu/Milan-1 & CIMMYT & $S$ & $1 \mathrm{~B} / 1 \mathrm{R}$ & $P m 8+?$ & 4 & 0 & 4 & 4 & 4 & 4 & 4 & 4 & 0 & 4 & 0 & 0 & 4 & 4 & 0 & 4 & 0 & 0 & 0 & 4 \\
\hline Tnmu/Milan-2 & CIMMYT & S & $1 \mathrm{~B} / 1 \mathrm{R}$ & $P m 3 d+?$ & 4 & 0 & 0 & 4 & 4 & 4 & 4 & 4 & 4 & 4 & 0 & 4 & 4 & 4 & 1 & 2 & 1 & 3 & 4 & \\
\hline Tnmu/Milan-3 & CIMMYT & $\mathrm{S}$ & $1 \mathrm{~B} / 1 \mathrm{R}$ & Pm8+? & 4 & 3 & 0 & 4 & 4 & 4 & 4 & 4 & 4 & 4 & 4 & 4 & 4 & 4 & 0 & 4 & 0 & 3 & 0 & 4 \\
\hline Tnmu/Milan-4 & CIMMYT & $\mathrm{S}$ & $1 \mathrm{~B} / 1 \mathrm{R}$ & Pm8+? & 4 & 4 & 4 & 4 & 4 & 4 & 4 & 4 & 4 & 4 & 4 & 4 & 4 & 4 & 0 & 4 & 0 & 4 & 0 & 4 \\
\hline Tx71A1039.V1*3/Ami//Trap\#1 & CIMMYT & W & $1 \mathrm{~B} / 1 \mathrm{R}$ & $P m 3 d+6+?$ & 4 & 0 & 0 & 4 & 4 & 4 & 4 & 4 & 0 & 4 & 0 & 3 & 3 & 3 & 0 & 2 & 4 & 0 & 4 & r \\
\hline Tx71A1039.V1*3/Ami//Turaco & CIMMYT & $\mathrm{W}$ & $1 \mathrm{~B} / 1 \mathrm{~B}$ & $?$ & 4 & 0 & 4 & 4 & 4 & 4 & 0 & 4 & 0 & 4 & 2 & 3 & 4 & 4 & 0 & 4 & 3 & 0 & 0 & 0 \\
\hline Vee/Koel//He1/3*Cno79/ 3/ Kauz & CIMMYT & $\mathrm{S}$ & $1 \mathrm{~B} / 1 \mathrm{R}$ & $P m 8+3 d+?$ & 4 & 0 & 0 & 4 & 4 & 4 & 4 & 4 & 4 & 4 & 0 & 3 & 0 & 4 & 0 & 2 & 4 & 3 & 0 &  \\
\hline Yang87-158/Ducula & CIMMYT & S & $1 \mathrm{~B} / 1 \mathrm{~B}$ & $?$ & 4 & 0 & 4 & 4 & 4 & 4 & 4 & 4 & 4 & 4 & 4 & 0 & 4 & 4 & 4 & 4 & 4 & 3 & 4 & 4 \\
\hline $\mathrm{Br} 23 / \mathrm{Emb} 40$ & Brazil & S & $1 \mathrm{~B} / 1 \mathrm{R}$ & Pm8+? & 4 & 0 & 0 & 4 & 4 & 4 & 4 & 4 & 4 & 4 & 4 & 0 & 3 & 4 & 0 & 4 & 0 & 0 & 0 & 4 \\
\hline Chat/Cep7780//Prl/Bow & Brazil & S & $1 \mathrm{~B} / 1 \mathrm{R}$ & $?$ & 4 & 4 & 4 & 4 & 4 & 4 & 4 & 4 & 4 & 4 & 4 & 2 & 4 & 4 & 4 & 4 & 4 & 4 & 0 & 4 \\
\hline Embra & Brazil & $\mathrm{S}$ & $\ldots$ & $?$ & 4 & 4 & 0 & 4 & 4 & 4 & 4 & 4 & 4 & 0 & 4 & 3 & 4 & 4 & 4 & 4 & 4 & 3 & 4 & 4 \\
\hline Jur/2*Iapar29//Pf8 & Brazil & S & $1 \mathrm{~B} / 1 \mathrm{R}$ & $\operatorname{Pm} 8$ & 4 & 4 & 4 & 4 & 4 & 4 & 4 & 4 & 4 & 4 & 4 & 4 & 4 & 4 & 0 & 4 & 3 & 4 & 0 & 4 \\
\hline Nd610/Kauz//Tui & Brazil & S & $1 \mathrm{~B} / 1 \mathrm{R}$ & Pm8+? & 0 & 0 & 0 & 0 & 0 & 0 & 0 & 4 & 0 & 3 & 0 & 4 & 4 & 4 & 0 & 0 & 0 & 3 & 0 & \\
\hline Stozher & Bulgaria & $\mathrm{W}$ & $1 \mathrm{~B} / 1 \mathrm{~B}$ & $?$ & 4 & 0 & 4 & 3 & 4 & 4 & 4 & 4 & 0 & 4 & 0 & 3 & 4 & 4 & 2 & 4 & 4 & 3 & 0 & 3 \\
\hline Soissons-1 & France & W & $1 \mathrm{~B} / 1 \mathrm{~B}^{*}$ & $?$ & 4 & 0 & 4 & 3 & 4 & 3 & 4 & 4 & 3 & 4 & 4 & 4 & 4 & 4 & 4 & 4 & 3 & 3 & 4 & 4 \\
\hline Soissons-2 & France & W & $1 \mathrm{~B} / 1 \mathrm{~B}^{*}$ & $P m 3 d+?$ & 4 & 0 & 4 & 4 & 4 & 3 & 0 & 4 & 3 & 4 & 0 & 4 & 4 & 3 & 4 & 2 & 4 & 3 & 4 & 4 \\
\hline Soissons-3 & France & W & 1B/1B* & $P m 3 d+?$ & 4 & 0 & 4 & 3 & 4 & 3 & 4 & 4 & 3 & 4 & 0 & 4 & 4 & 4 & 4 & 2 & 4 & 3 & 4 & T \\
\hline Contra & Germany & W & $1 \mathrm{~B} / 1 \mathrm{~B}^{*}$ & $P m 4 b+?$ & 0 & 0 & 0 & - & 0 & 0 & 0 & 0 & 0 & 0 & 0 & 4 & 4 & 4 & 0 & 0 & 0 & 0 & 0 & 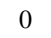 \\
\hline German 8 & Germany & W & $\ldots$ & $P m 4 b$ & 1 & 0 & 0 & 2 & 0 & 0 & 0 & 0 & 1 & 3 & 0 & 4 & 4 & 4 & 0 & 3 & 0 & 0 & 0 & 0 ; \\
\hline Mv Madrigal & Hungary & W & $1 \mathrm{~B} / 1 \mathrm{R}$ & Pm8+? & 4 & 4 & 4 & 4 & 4 & 4 & 4 & 4 & 4 & 4 & 4 & 4 & 4 & 4 & 2 & 4 & 1 & 4 & 0 & , \\
\hline Mv Magdalena & Hungary & $\mathrm{W}$ & $1 \mathrm{~B} / 1 \mathrm{R}$ & Pm8+? & 4 & 4 & 4 & 4 & 4 & 4 & 4 & 4 & 4 & 4 & 4 & 4 & 4 & 4 & 1 & 4 & 1 & 4 & 0 & 4 \\
\hline Mv Optima & Hungary & W & $1 \mathrm{~B} / 1 \mathrm{R}$ & $\operatorname{Pm} 8$ & 4 & 4 & 4 & 4 & 4 & 4 & 4 & 4 & 4 & 4 & 4 & 4 & 4 & 4 & 1 & 4 & 4 & 4 & 0 & 4 \\
\hline $\mathrm{Mv}-17$ & Hungary & W & $\ldots$ & $?$ & 0 & 0 & 0 & 0 & 0 & 0 & 0 & 0 & 0 & 0 & 0 & 0 & 0 & 0 & 0 & 0 & 0 & 0 & 0 & 0 \\
\hline $\mathrm{Mv}-23$ & Hungary & W & $1 \mathrm{~B} / 1 \mathrm{R}$ & $?$ & 4 & 4 & 4 & 4 & 4 & 4 & 4 & 4 & 4 & 4 & 3 & 4 & 4 & 4 & 1 & 4 & 4 & 4 & 3 & 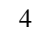 \\
\hline Campion & Romania & W & $1 \mathrm{~B} / 1 \mathrm{~B}$ & Pm $3 a+6+?$ & 0 & 0 & 0 & 0 & 4 & 4 & 3 & 4 & 0 & 3 & 0 & 0 & 0 & 3 & 2 & 4 & 4 & 0 & 3 & 4 \\
\hline Rah122/94 & Romania & $\mathrm{W}$ & $\ldots$ & $P m 4 a$ & 0 & 4 & 0 & 0 & 0 & 0 & 3 & 0 & 4 & 4 & 4 & 4 & 3 & 4 & 0 & 0 & 1 & 2 & 0 & 0 \\
\hline Lona & Switzerland & $\mathrm{S}$ & $1 \mathrm{~B} / 1 \mathrm{~B}^{*}$ & $?$ & 4 & 4 & 4 & 4 & 4 & 0 & 4 & 4 & 4 & 3 & 4 & 4 & 4 & 4 & 0 & 4 & 4 & 4 & 4 & 4 \\
\hline Hussar & United Kingdom & S & $1 \mathrm{~B} / 1 \mathrm{R}^{*}$ & Pm8+? & 0 & 0 & 4 & 0 & 0 & 0 & 0 & 0 & 0 & 0 & 0 & 4 & 4 & 4 & 0 & 0 & 0 & 0 & 0 & 0 ; \\
\hline Cty*3/Ta2450 & United States & $\mathrm{W}$ & $1 \mathrm{~B} / 1 \mathrm{R}$ & Pm8+? & 4 & 0 & 3 & 3 & 4 & 4 & 0 & 4 & 0 & 4 & 2 & 3 & 3 & 3 & 0 & 1 & 2 & 0 & 0 & 0 \\
\hline Massey & United States & W & $1 \mathrm{~B} / 1 \mathrm{~B}$ & $?$ & 4 & 0 & 4 & 3 & 4 & 4 & 4 & 4 & 4 & 0 & 4 & 4 & 4 & 3 & 4 & 3 & 4 & 3 & 4 & 0 \\
\hline Va91-51-26 & United States & W & $\ldots$ & $?$ & 0 & 0 & 0 & 0 & 0 & 0 & 0 & 0 & 0 & 0 & 0 & 0 & 0 & 0 & 0 & 0 & 0 & 0 & 0 & 0 \\
\hline Va91-52-65 & United States & W & $\ldots$ & $?$ & 0 & 0 & 0 & 0 & 0 & 0 & 0 & 0 & 0 & 0 & 0 & 0 & 0 & 0 & 0 & 0 & 0 & 0 & 0 & 0 \\
\hline 90-I145 & $\ldots$ & W & $\ldots$ & $?$ & 4 & 0 & 4 & 4 & 4 & 4 & 4 & 4 & 4 & 4 & 3 & 4 & 2 & 4 & 0 & 4 & 3 & 0 & 3 & 4 \\
\hline 90-I89 & $\ldots$ & W & $\ldots$ & $P m 3 d$ & 4 & 4 & 4 & 4 & 4 & 4 & 4 & 4 & 4 & 4 & 0 & 4 & 4 & 4 & 4 & 0 & 4 & 4 & 4 & 4 \\
\hline Sxafa4-3 & $\ldots$ & W & $1 \mathrm{~B} / 1 \mathrm{~B}$ & $P m 3 f+?$ & 4 & 4 & 0 & 0 & 0 & 0 & 4 & 0 & 4 & 4 & 3 & 4 & 4 & 4 & 0 & 0 & 0 & 0 & 4 & ; \\
\hline
\end{tabular}


Table 4. Analysis of variance of relative area under disease progress curve (AUDPC) for disease index and maximum disease severity (MDS) on penultimate leaf (F-1) in 52 and 76 wheat cultivars or lines tested in the 2000-01 and 2002-03 crop seasons, respectively

\begin{tabular}{llrcc}
\hline Season, parameter & Source of variation & df & Mean of squares & $\boldsymbol{F}$ value $^{\mathbf{a}}$ \\
\hline 2000-01 & & & & $528.6^{* *}$ \\
AUDPC & Genotypes & 51 & 0.2643 & $<1.0$ \\
& Replicates & 2 & 0.0003 & $\ldots$ \\
Error & 102 & 0.0005 & $242.6^{* *}$ \\
MDS & & & $<1.0$ \\
& Genotypes & 51 & 315.673 & $\ldots$ \\
Replicates & 2 & 0.427 & $137.0^{* *}$ \\
AUDPC & Error & 102 & 1.301 & $<1.0$ \\
& Genotypes & 75 & 0.1233 & $\ldots$ \\
MDS & Replicates & 2 & 0.0002 & $385.2^{* *}$ \\
& Error & 150 & 0.0009 & $\ldots$ \\
\hline
\end{tabular}

a $* *$ Indicates significant difference at $P=0.01$.

Table 5. Twenty-two wheat cultivars and lines with adult plant resistance (APR) to powdery mildew identified in the 2000-01 and 2002-03 crop seasons $^{\mathrm{a}}$

\begin{tabular}{|c|c|c|c|c|c|}
\hline \multirow[b]{2}{*}{ Cultivar or line } & \multicolumn{2}{|c|}{$2000-01$} & \multicolumn{2}{|c|}{$2002-03$} & \multirow[b]{2}{*}{ Reaction $^{b}$} \\
\hline & AUDPC & $\operatorname{MDS}(\%)$ & AUDPC & $\operatorname{MDS}(\%)$ & \\
\hline 98301 & $\ldots$ & $\ldots$ & 0.04 & 2.2 & 4 \\
\hline 8030 Versailles/Edch//Cd & $\ldots$ & $\ldots$ & 0.04 & 2.5 & 4 \\
\hline Arina & $\ldots$ & $\ldots$ & 0.02 & 1.0 & 4 \\
\hline Bainong 64 & 0.08 & 3.0 & 0.05 & 3.4 & 4 \\
\hline CA8686 & $\ldots$ & $\ldots$ & 0.02 & 1.4 & 4 \\
\hline CA9550 & 0.03 & 4.0 & 0.04 & 2.0 & 4 \\
\hline CA9641 & 0.03 & 4.0 & 0.02 & 1.0 & 4 \\
\hline Hussar & 0.03 & 2.0 & 0.02 & 1.0 & 4 \\
\hline Lona & $\ldots$ & $\ldots$ & 0.03 & 1.3 & 4 \\
\hline Mv Madrigal & $\ldots$ & $\ldots$ & 0.03 & 1.7 & 4 \\
\hline Mv Magdalena & $\ldots$ & $\ldots$ & 0.04 & 2.3 & 4 \\
\hline Mv-23 & 0.05 & 6.8 & 0.01 & 0.8 & 4 \\
\hline Shandong 418 & $\ldots$ & $\ldots$ & 0.02 & 1.4 & 4 \\
\hline Soissons-1 & $\ldots$ & $\ldots$ & 0.04 & 2.8 & 4 \\
\hline Soissons-2 & $\ldots$ & $\ldots$ & 0.03 & 1.7 & 4 \\
\hline Soissons-3 & $\ldots$ & $\ldots$ & 0.03 & 1.5 & 4 \\
\hline Sxafa4-3 & 0.04 & 4.0 & 0.00 & 0.0 & 4 \\
\hline Xingmai 99 & 0.03 & 3.0 & 0.02 & 1.5 & 4 \\
\hline YE-2416-7A & 0.03 & 4.0 & 0.00 & 0.0 & 4 \\
\hline Yumai47 & $\ldots$ & $\ldots$ & 0.04 & 2.5 & 4 \\
\hline Yuzhan9705 & $\ldots$ & $\ldots$ & 0.03 & 1.7 & 4 \\
\hline Zhoumai 17 & $\ldots$ & $\ldots$ & 0.03 & 1.9 & 4 \\
\hline Alidos ${ }^{\mathrm{c}}$ & 0.05 & 5.0 & 0.07 & 3.1 & 4 \\
\hline Andros ${ }^{c}$ & 0.06 & 6.0 & 0.05 & 3.0 & 4 \\
\hline FAP77517c & 0.06 & 5.0 & 0.04 & 2.0 & 4 \\
\hline Massey $^{\mathrm{c}}$ & 0.04 & 6.0 & 0.00 & 0.6 & 4 \\
\hline Mercia $^{c}$ & 0.06 & 7.0 & 0.02 & 1.4 & 4 \\
\hline Redcoat $^{\mathrm{c}}$ & 0.06 & 5.0 & 0.02 & 1.0 & 4 \\
\hline Jingshuang $16^{\mathrm{d}}$ & 1.00 & 92.0 & 1.00 & 95.0 & 4 \\
\hline
\end{tabular}

a AUDPC = relative area under disease progress curve for disease index for cultivar or line divided by AUDPC for susceptible check Jingshuang $16 ; \ldots=$ not tested; MDS = maximum disease severity.

b Seedling reaction to E20; 4 = colonies with well-developed hyphae and abundant conidia, and colonies mostly joined together.

${ }^{\mathrm{c}}$ Resistant check cultivar with known APR to powdery mildew.

d Susceptible check cultivar. translocation, as shown in Table 3. However, $71.5 \%$ of $1 \mathrm{~B} / 1 \mathrm{R}$ translocation lines did not show the reaction pattern expected for $P m 8$ (Table 3), which might be due to the presence of suppressor genes for $\operatorname{Pm} 8$ (25). This was consistent with the results of a previous study by Ren et al. (25), who found that, on average, only one-third of all CIMMYT wheats with $1 \mathrm{~B} / 1 \mathrm{R}$ tested actually expressed the $P m 8$ gene.
There is an urgent need for a better genetic strategy for powdery mildew control. Several strategies have been proposed for developing durable resistance to powdery mildew, including the pyramiding of effective major resistance genes (32), the use of multilines with different resistance genes (3), and breeding cultivars with APR or slow mildewing resistance (28). The use of molecular markers to pyramid major genes seemed promising (22), but may cause the rapid formation of isolates with multiple matching virulence (10). Therefore, APR has been suggested as the most sustainable strategy for breeding wheat cultivars with durable powdery mildew resistance $(1,28)$.

A series of studies on APR to powdery mildew were conducted during the last decade, and both qualitative and quantitative genes were detected in various wheat cultivars $(4,5,9)$. Chae and Fischbeck (4) reported the effect of 14 chromosomes on APR to powdery mildew in cv. Diplomat; whereas, in cvs. Knox 62, Massey, Redcoat, and Houser, APR is governed by two to three genes with moderate to high heritability $(5,9)$. CIMMYT has conducted several studies on slow rusting and found that combinations of four to five minor genes for APR to leaf rust and stripe rust give adequate protection in the field (30).

Bainong 64, a leading cultivar in China until 2000, was grown on about 700,000 ha annually for 8 years. Our study confirmed that it carries APR (Fig. 1). This cultivar was used widely as a parent, and we are testing several derived lines for possible release in the Huang-Huai winter wheat zone of China.

Description of APR can be carried out based on different parameters, such as a disease index, AUDPC, and MDS on penultimate leaf $(11,36)$. In the present study, we used AUDPC, MDS, and a disease index to evaluate APR in the field. All cultivars or lines with APR showed lower values for AUDPC, MDS, and the disease index than susceptible cultivars (Table 5). In practical breeding, it is good to use a simple parameter to evaluate APR in the field. Investigations of AUDPC and the disease index are time-consuming and, because AUDPC and MDS are highly correlated $(r=0.96, P<0.001)$, it is more practical to use the latter parameter for powdery mildew screening, with a single scoring at an appropriate time.

Generally, APR to powdery mildew is detected in cultivars that either have no identified major resistance genes or whose major-gene resistance has been overcome (1). As an example, most powdery mildew populations worldwide show high virulence frequencies for $P m 5$ at the seedling stage (14), but the gene serves as a source of APR $(14,20)$. In our study, 17 cultivars and lines whose race-specific resistance genes were overcome in the seedling stage by isolate E20 showed APR in the field, indicating that they might be carrying mi-
36 cultivars and lines due to the lack of $B$. graminis f. sp. tritici isolates or differential lines (Table 3). In addition, 94 genotypes were susceptible to all $B$. graminis f. sp. tritici isolates (Table 2), which indicates that they either carry no resistance genes or possess resistance genes that were overcome by matching virulence in all 20 B. graminis $\mathrm{f}$. $\mathrm{sp}$. tritici isolates used in this study. 


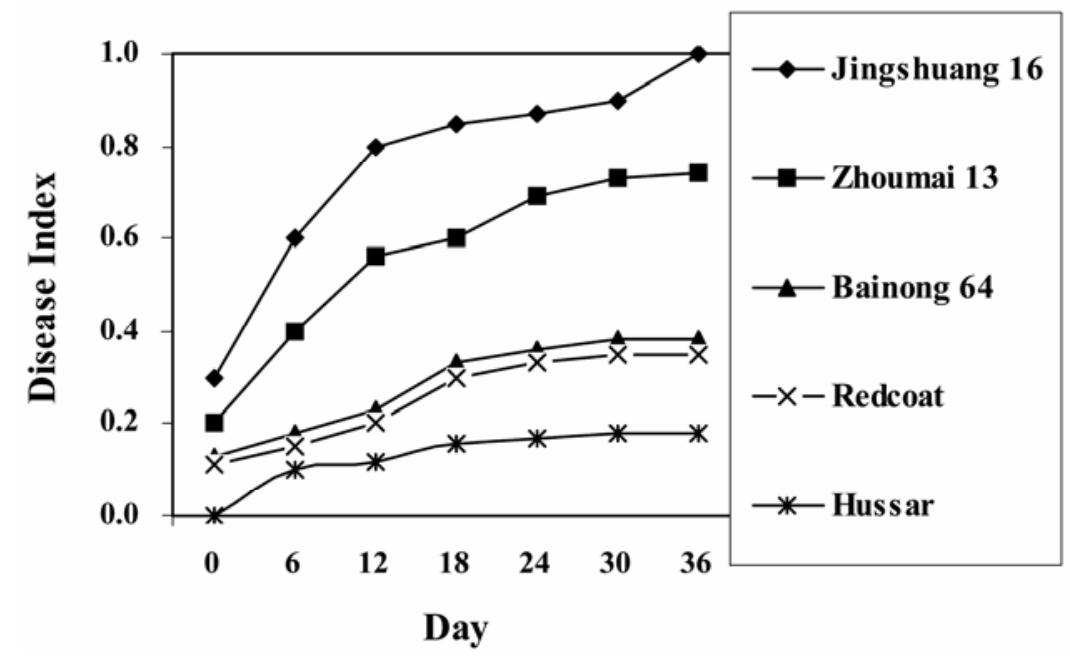

Fig. 1. Progress curve of powdery mildew development in wheat based on the index of disease at seven scoring dates (indicated as cumulative days; day $0=4$ weeks after inoculation). Jingshuang 16 is a highly susceptible cultivar, Zhoumai 13 is a moderately susceptible cultivar, Bainong 64 is a Chinese cultivar with adult plant resistance (APR), Redcoat is a US cultivar with known APR, and Hussar is a UK cultivar with APR.

nor APR genes in addition to the major genes (Tables 3 and 5). Results indicate that it is possible to combine major resistance genes and APR genes to achieve durable resistance to powdery mildew in wheat cultivars.

\section{ACKNOWLEDGMENTS}

We thank X. Huang for his critical review of this paper and A. McNab for editing it. This project is supported by the National Natural Science Foundation of China (30220140636).

\section{LITERATURE CITED}

1. Bennett, F. G. A. 1984. Resistance to powdery mildew in wheat: a review of its use in agriculture and breeding programmes. Plant Pathol. 33:279-300.

2. Bjarko, M. E., and Line, R. F. 1988. Heritability and number of genes controlling leaf rust resistance in four cultivars of wheat. Phytopathology 78:457-461.

3. Browning, J. A., and Frey, K. J. 1969. Multiline cultivars as a means of disease control. Annu. Rev. Phytopathol. 7:355-382.

4. Chae, Y. A., and Fischbeck, G. W. 1979. Genetic analysis of powdery mildew resistance in wheat cultivar "Diplomat". Z. Pflanzenzeucht. 83:272-280.

5. Das, M. K., and Griffey, C. A. 1994. Heritability and number of genes governing adult-plant resistance to powdery mildew in Houser and Redcoat winter wheats. Phytopathology 84:406-409.

6. Duan, X. Y., and Sheng, B. Q. 1998. Identification of isolates of Blumeria graminis $\mathrm{f}$. $\mathrm{sp}$. tritici and the monitoring of their virulence frequencies. Acta Phytopathol. Sin. 25:31-36.

7. Duan, X. Y., Xiang, Q. J., Zhou, Y. L., and Sheng, B. Q. 1993. Establishment of wheat powdery mildew isolates with putative virulence genotype. Plant Prot. 19:27-28.

8. Fried, P. M., Mackenzie, D. R., and Nelson, R. R. 1981. Yield loss caused by Erysiphe graminis f. sp. tritici on single culms of "Chancellor" wheat and four multilines $\mathrm{Z}$. Pflanzenkrankh. Pflanzenschutz 88:256-264.

9. Griffey, C. A., and Das, M. K. 1994. Inheritance of adult-plant resistance to powdery mildew in Knox 62 and Massey winter wheats. Crop Sci. 34:641-646.

10. Groth, J. V. 1976. Multilines and "superraces": a simple model. Phytopathology 66:937-939.

11. Guo, Y. Z., Duan, S. K., and Zhang, T. 1999. A study of classifying resistant types to scab in wheat with the index of disease severity. Acta Agric. Univ. Henanensis 33:336-339. Identification of rye chromosome 1R translocation and subunits in hexaploid wheat using storage proteins as genetic markers. Plant Breed. 109:130-140.

13. He, Z. H., Rajaram, S., Xin, Z. Y., and Zhang, G. Z., eds. 2001. A History of Wheat Breeding in China. CIMMYT, Mexico, D.F.

14. Hsam, S. L. K., and Zeller, F. J. 2002. Breeding for powdery mildew resistance in common wheat (Triticum aestivum L.). Pages 219-238 in: The Powdery Mildews: A Comprehensive Treatise. R. Belanger, B. Bushnell, A. Dik, and T. Carver, eds. The American Phytopathological Society, St. Paul, MN.

15. Huang, X. Q., Hsam, S. L. K., and Zeller, F. J. 1997. Identification of powdery mildew resistance genes in common wheat (Triticum aestivum L. em Thell). 9. Cultivars, land races and breeding lines grown in China. Plant Breed. 116:233-238.

16. Huang, X. Q., and Röder, M. S. 2004. Molecular mapping of powdery mildew resistance genes in wheat: A review. Euphytica 137:203223.

17. Kazman, M. E., and Lein, V. 1996. Cytological and SDS-PAGE characterization of 1994-95grown European wheat cultivars. Annu. Wheat Newsl. 42:86-92.

18. Leath, S., and Bowen, K. L. 1989. Effects of powdery mildew, triadimenol seed treatment
12. Gupta, R. B., and Shepherd, K. W. 1992. and triadimefon foliar sprays on yield of winter wheat in North Carolina. Phytopathology 79:152-155.

19. Leath, S., and Heun, M. 1990. Identification of powdery mildew resistance genes in cultivars of soft winter wheat. Plant Dis. 74:747-752.

20. Lebsock, K. L., and Briggle, L. W. 1974. Gene Pm5 for resistance to Erysiphe graminis f. sp. tritici in Hope wheat. Crop Sci. 14:561-563.

21. Lipps, P. E., and Madden, L. V. 1988. Effect of triadimenol seed treatment and triadimefon foliar treatment on powdery mildew epidemics and grain yield of winter wheat cultivars. Plant Dis. 72:887-892.

22. Liu, J., Liu, D., Tao, W., Li, W., Wang, S. Chen, P., Cheng, S., and Gao, D. 2000. Molecular marker-facilitated pyramiding of different genes for powdery mildew resistance in wheat. Plant Breed. 119:21-24.

23. Liu, X. K. 1989. Prospects of wheat powdery mildew research in China. Inf. Agric. Husb. August 1989:1-10.

24. McIntosh, R. A., Yamazaki, Y., Devos, K. M., Dubcovsky, J., Rogers, W. J. and Appels, R., 2003: Catalogue of gene symbols for wheat. Pages 1-34 in: Proc. 10th Int. Wheat Genet. Symp. Vol. 4.

25. Ren, S. X., McIntosh, R. A., and Lu, Z. J. 1997. Genetic suppression of the cereal rye-derived gene $P m 8$ in wheat. Euphytica 93:353360.

26. Saari, E. E., and Prescott, J. M. 1975. A scale for appraising the foliar intensity of wheat diseases. Plant Dis. Rep. 59:377-381.

27. Schlegel, R. 1997. Current list of wheats with rye introgressions of homoeologous group 1 . 2nd update. Wheat Inf. Serv. 84:64-69.

28. Shaner, G. 1973. Evaluation of slow-mildewing resistance of Knox wheat in the field. Phytopathology 63:867-872.

29. Shi, Q. M., Zhang, X. X., and Duan, X. Y. 1987. Identification of isolates of Blumeria graminis f. sp. tritici. Sci. Agric. Sin. 20:6470 .

30. Singh, R. P., Huerta-Espino, J., and William, M. 2001. Slow rusting genes based resistance to leaf and yellow rusts in wheat. Pages 103108 in: Proc. 10th Assembly Wheat Breed. Soc. Aust. Inc. R. Eastwood, G. Hollamby, T. Rathjen, and N. Gororo, eds. Mildura, Australia.

31. Tao, J. F., Shen, X. Z., Qin, J. Z., and Qin, Y. 1982. Studies on the resistance to powdery mildew in wheat species and varieties. Acta Phytopathol. Sin. 12:7-14.

32. Vanderplank, J. E. 1984. Disease Resistance in Plant, 2nd ed. Academic Press, New York.

33. Wu, Z. S. 1990. Breeding for wheat disease resistance. Pages 235-272 in: Wheat Breeding. Z. S. Wu, ed. Agric. Pub. Press China, Beijing.

34. Xia, X. C., Hasm, S. L. K., Stephan, U., Yang, T. M., and Zeller, F. J. 1995. Identification of powdery mildew resistance genes in common wheat (Triticum aestivum L). VI. Wheat cultivars grown in China. Plant Breed. 114:174 175.

35. Xiang, Q. J., Sheng, B. Q., Zhong, Y. L., Duan, X. Y., and Zhang, K. C. 1994. Analyses of resistance genes of three differential varieties to the isolates of Blumeria graminis f. sp. tritici in wheat. Race. Acta Agric. Boreali-Sin. 9:94-97.

36. Yu, D. Z., Yang, X. J., Yang, L. J., Jeger, M. J., and Brown, J. K. M. 2001. Assessment of partial resistance to powdery mildew in Chinese wheat varieties. Plant Breed. 120:279-284. 